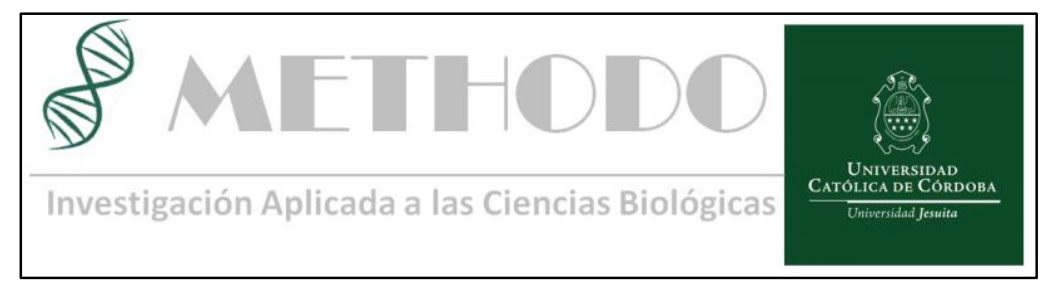

BIOESTADISTICA Y METODOLOGIA APLICADA Methodo 2017 Sep;2(3): 99-105 DOI: $10.22529 / \mathrm{me} .2017 .2(3) 05$

\title{
¿El estudio radiográfico de senos paranasales es útil para confirmar el diagnóstico de sinusitis aguda? ${ }^{1}$
}

\section{Is the radiographic study of paranasal sinuses useful to confirm the diagnosis of acute sinusitis?}

1. Ateneo Central. Facultad de Ciencias de la Salud. Universidad Católica de Córdoba. 14 de junio de 2017. Actividad desarrollada por la Secretaría de Investigación y la Cátedra de Bioestadística de la Carrera de Medicina

Alegre $\mathrm{G}^{1}$, Azar $\mathrm{T}^{2}$, Marelli $\mathrm{S}^{2}$, Pfleger $\mathrm{M}^{2}$, Revol $\mathrm{S}^{2}$, Cuestas $\mathrm{E}^{3}$.

\section{Resumen}

INTRODUCCIÓN: La sinusitis es una patología frecuente en la práctica clínica; se considera que entre un 5 y un $10 \%$ de las infecciones de la vía aérea superior se complican con infección de los senos paranasales. Considerando que el promedio de las infecciones respiratorias anuales en los niños oscila entre los 6 y 8 episodios se estima que la incidencia de sinusitis es elevada, aunque difícil de precisar. Por cuestiones anatómicas y funcionales hay mayor incidencia de esta afección durante la infancia. Por sinusitis se entiende la inflamación de origen infeccioso de la mucosa de los senos paranasales, y su diagnóstico es clínico.

METODOLOGíA: Desde la exposición de un escenario clínico se planteó una pregunta estructurada para luego realizar una búsqueda bibliográfica con el fin de dar respuesta sobre la necesidad del uso de estudios radiográficos de los senos paranasales para el diagnóstico de la sinusitis aguda en niños. Se realizó búsqueda en PubMed utilizando los Mesh (Medical Subject Headings) "sinusitis AND radiography" y aplicando distintos filtros, tras lo cual se encontró sólo un meta-análisis y, consultado la bibliografía citada por éste, se hayo un único estudio específico para población pediátrica.

ANÁLISIS DEL ARTíCULO: El diseño del trabajo no es específico para un estudio de diagnóstico, por lo que para el análisis de la validez estadística del artículo debimos realizar un análisis secundario de los datos del mismo con el fin de definir los valores de sensibilidad, especificidad, valor predictivo positivo y valor predictivo negativo, con sus respectivos IC95\%, a fin de aproximarnos a una conclusión más confiable. Dentro de estos resultados se obtuvo una sensibilidad del 76,04\% con un intervalo de confianza del 95\% (IC $95 \%$ ) de 66,61 $83,47 \%$, y una especificidad de $69,62 \%$ (IC $95 \%$ de $58,77-78,66 \%$ ).

RECOMENDACIÓN FINAL: Por lo tanto, no se recomienda realizar radiografías de senos paranasales en niños con episodios de sinusitis. El diagnóstico de sinusitis aguda en niños se hace sobre la base de criterios clínicos estrictos que describen signos, síntomas y patrones temporales de una infección de la vía respiratoria alta.

Palabras claves: SINUSITIS, DIAGNÓSTICO POR IMÁGENES, ESTADÍSTICA, INFANCIA.

Revista Methodo: Investigación Aplicada a las Ciencias Biológicas. Facultad de Ciencias de la Salud. Universidad Católica de Córdoba. Jacinto Ríos 571 Bo Gral. Paz. X5004FXS. Córdoba. Argentina. Tel.: (54) 3514517299 / Correo: methodo@ucc.edu.ar / Web: methodo.ucc.edu.ar | BIOESTADISTICA Y METODOLOGIA APLICADA Methodo 2017 Sep;2(3): 99-105 
Alegre G, Azar T, Marelli S, Pfleger M, Revol S, Cuestas E ¿El estudio radiográfico de senos paranasales es útil para confirmar el diagnóstico de sinusitis aguda?

\title{
Abstract
}

INTRODUCTION: Sinusitis is a frequent pathology in clinical practice. Between 5 and $10 \%$ of infections of the upper airway are complicated by acute sinusitis. Considering that the average annual catarrh in children ranges from 6 to 8 episodes, it is estimated that the incidence of sinusitis is high, although difficult to specify. There is a higher incidence of this condition in childhood for anatomical and functional reasons. The diagnosis of sinusitis, understood as infectious inflammation of the paranasal sinuses, is clinical.

METHODOLOGY: Since the presentation of a clinical situation, a structured question was asked to perform a bibliographic search in order to answer the question about the need of radiographic studies for the diagnosis of acute sinusitis in children. A PubMed search was performed using the Mesh (Medical Subject Headings) "sinusitis AND radiography" and applying different filters, after which only a meta-analysis was found and, with reference to the bibliography cited by the latter, there was a single specific study for childhood.

ANALYSIS OF THE ARTICLE: The work design is not specific to a diagnostic study. For the analysis of the statistical validity of the article, in order to approach a more reliable conclusion, we had to perform a secondary analysis of the data to define the values of sensitivity, specificity, positive predictive value and negative predictive value, with their respective $95 \%$ confidence interval $(95 \% \mathrm{Cl})$. As a result we obtained a sensitivity of $76,04 \%$ with a $95 \% \mathrm{Cl}$ of 66,61 $83,47 \%$, and a specificity of $69.62 \%$ with a $95 \% \mathrm{Cl}$ of $58,77-78,66 \%$.

RECOMMENDATION: Therefore, it is not necessary to perform radiography imaging in children with episodes of clinical sinusitis. Acute sinusitis in children is a diagnosis that is made on the basis of stringent clinical criteria that describe signs, symptoms, and temporal patterns of an upper respiratory tract infection

Key words: SINUSITIS, DIAGNOSIS IMAGING, STATISTICS, CHILDHOOD.

1. Docente Universidad Católica de Córdoba, Argentina, Facultad de Ciencias de la Salud, Cátedra de Inmunología. Clínica Universitaria Reina Fabiola, Servicio de Alergia e Inmunología.

2. Estudiantes de la Práctica Final Obligatoria. Universidad Católica de Córdoba, Argentina, Facultad de Ciencias de la Salud, Carrera de Medicina.

3. Profesor Titular. Universidad Católica de Córdoba, Argentina, Facultad de Ciencias de la Salud, Cátedra de Bioestadística.

4. Correspondencia: Graciela Alegre. Servicio de Alergia e Inmunología- Clínica Universitaria Reina Fabiola. Oncativo 1248 -X5004FHP- Córdoba, Argentina. e-mail: graalegre98@gmail.com

\section{INTRODUCCIÓN ESCENARIO CLÍNICO}

Además refiere cefalea leve en los últimos tres días. Inicialmente

Un niño de 10 años de edad, previamente sano, es traído a la consulta en compañía de su madre.

Presenta hace dos semanas congestión nasal, rinorrea $y$ tos productiva, acompañado de fiebre de $38-38,5^{\circ} \mathrm{C}$.

\author{
fue tratado con descongestivos y \\ antitérmicos (dipirona) para el control de \\ los síntomas, pero con escasa \\ respuesta. Al examen físico no se \\ constata fiebre, pero se evidencia
}

Revista Methodo: Investigación Aplicada a las Ciencias Biológicas. Facultad de Ciencias de la Salud. Universidad Católica de Córdoba. Jacinto Ríos 571 Bo Gral. Paz. X5004FXS. Córdoba. Argentina. Tel.: (54) 3514517299 / Correo: methodo@ucc.edu.ar / Web: methodo.ucc.edu.ar | DOI: 10.22529/me.2017.2(3)05 
Alegre G, Azar T, Marelli S, Pfleger M, Revol S, Cuestas E ¿El estudio radiográfico de senos paranasales es útil para confirmar el diagnóstico de sinusitis aguda?

secreción nasal mucopurulenta y dolor a

la compresión de los senos

paranasales. El médico pediatra que lo asiste en la guardia, ante la sospecha de sinusitis aguda, solicita radiografías de senos paranasales (mentonasoplaca y frontonasoplaca), tras lo cual le indica tratamiento con Amoxicilina durante 14 días por vía oral. Frente a esta situación, un alumno de la carrera de grado de Medicina, que estaba presente al momento de la consulta se cuestiona sobre la real utilidad diagnóstica de las pruebas radiográficas cuando el paciente presenta parámetros clínicos para el diagnóstico de sinusitis aguda.

\section{PREGUNTA CLÍNICA}

\section{ESTRUCTURADA}

¿En niños con sintomatología altamente sugestiva de sinusitis aguda [pacientes], son necesarios los estudios complementarios radiográficos de los senos paranasales [intervención] para definir el diagnóstico [resultado]?

\section{CONTEXTO CLÍNICO}

La sinusitis es una patología frecuente en la práctica clínica; se considera que entre un 5 y un $10 \%$ de las infecciones de la vía aérea superior se complican con una sinusitis aguda. Considerando que la media de catarros anuales en los niños oscila entre los 6 y 8 episodios, se estima que la incidencia de sinusitis es elevada, aunque difícil de precisar ${ }^{(1)}$.

En los niños, los senos son más pequeños, y sus ostium también. Esto puede justificar la mayor incidencia de esta afección en la infancia y explicaría, así mismo, la mayor frecuencia de participación de los senos anteriores, ventilados por el ostium de menor tamaño. A pesar de tratarse de una enfermedad frecuente, en muchas ocasiones la sinusitis no se diagnostica, debido a la dificultad para diferenciarla del proceso catarral que habitualmente la precede ${ }^{(1)}$

Por sinusitis se entiende la inflamación de origen infeccioso de la mucosa de los senos paranasales $y$, en ocasiones, de las paredes óseas que la rodean y sobre las que asienta ésta. El diagnóstico de la sinusitis aguda es clínico. En la exploración física encontraremos rinorrea purulenta anterior y posterior, así como congestión de la mucosa de ambas fosas nasales, hallazgos inespecíficos que también aparecen en la rinitis aguda. La hipersensibilidad local, el edema periorbitario o la halitosis son muy sugestivos de sinusitis, siempre que aparezcan en el contexto de los síntomas respiratorios descritos con anterioridad. La visualización de pus drenando desde el meato medio es diagnóstica de sinusitis, pero un niño difícilmente permite ser explorado con la exhaustividad necesaria para confirmar este dato ${ }^{(1)}$.

La complicación más común de la sinusitis aguda en niños implica la órbita. Se deben sospechar

Revista Methodo: Investigación Aplicada a las Ciencias Biológicas. Facultad de Ciencias de la Salud.

Universidad Católica de Córdoba. Jacinto Ríos 571 Bo Gral. Paz. X5004FXS. Córdoba. Argentina. Tel.:

(54) 3514517299 / Correo: methodo@ucc.edu.ar / Web: methodo.ucc.edu.ar | DOI: 
Alegre G, Azar T, Marelli S, Pfleger M, Revol S, Cuestas E ¿El estudio radiográfico de senos paranasales es útil para confirmar el diagnóstico de sinusitis aguda?

complicaciones orbitarias cuando el niño presenta una tumoración ocular, especialmente si está acompañado de proptosis o deterioro de la función de los músculos extraoculares. Las complicaciones orbitarias de la sinusitis aguda se han dividido en 5 categorías: edema inflamatorio, absceso subperiosteal, celulitis orbitaria, absceso orbitario y trombosis del seno cavernoso. Éstas complicaciones se visualizan mejor mediante la Tomografía Computarizada (TC) contrastada. Se deben sospechar complicaciones intracraneales en el paciente que presenta un dolor de cabeza muy severo, fotofobia, convulsiones $u$ otros hallazgos neurológicos focales. Las complicaciones intracraneales incluyen el empiema subdural, el empiema epidural, la trombosis venosa, el absceso cerebral y la meningitis. En general, la TC con contraste ha sido el estudio de imagen preferido cuando se sospecha alguna de las complicaciones de la sinusitis ${ }^{(2)}$.

\section{ESTRATEGIA DE BÚSQUEDA}

Para responder a la pregunta se realizó una búsqueda en PubMed utilizando los Mesh (Medical Subject Headings) "sinusitis AND radiography". E resultado obtenido inicialmente fue de 4653 artículos, tras lo cual se aplicaron los siguientes filtros: en relación al tipo de artículo buscado, systematic reviews; y con respecto al rango etario, niños desde el nacimiento hasta los 18 años. El número de resultados disminuyó a 23 artículos, y luego de la lectura de los resúmenes, se encontró sólo un metaanálisis ${ }^{(3)}$; el cual tiene un nivel 1 de los grados de evidencias y, consultado la bibliografía citada por éste se encontró un único estudio específico para población pediátrica ${ }^{(4)}$ con un nivel 3 de los grados de evidencia, que nos permite responder a la pregunta planteada.

\section{RESUMEN DEL ARTÍCULO}

Este estudio prospectivo compara los síntomas, los hallazgos clínicos y bacteriológicos obtenidos por punción de los senos paranasales con los hallazgos radiológicos en un esfuerzo por encontrar una combinación plausible de síntomas y signos que permita un diagnóstico fiable de sinusitis aguda.

El grupo de estudio estaba compuesto por 175 niños que fueron investigados consecutivamente en un estudio prospectivo debido a la sospecha clínica de sinusitis aguda. Los senos paranasales fueron invariablemente examinados radiológicamente.

médico consultor rellenó un cuestionario referente a los antecedentes previos del paciente y los síntomas y signos clínicos en el momento de la consulta. Las historias incluyeron incidencias de enfermedad sinusal, otitis media 
Alegre G, Azar T, Marelli S, Pfleger M, Revol S, Cuestas E ¿El estudio radiográfico de senos paranasales es útil para confirmar el diagnóstico de sinusitis aguda?

recurrente con derrame, infección respiratoria alta (IRA), faringoamigdalitis, bronquitis, alergia, etc. El examen clínico incluyó un cuidadoso examen de los oidos, la nariz y la faringe.

El examen radiológico incluyó vistas occipito-mentales y occipito-frontales, una vista lateral y una vista occipitomental en la posición lateral, según lo descrito por Axelsson y Jensen ${ }^{(5)}$. Los hallazgos radiológicos (neumatización de los senos, edema de la mucosa, líquido, pólipos y quistes) se observaron en una forma especialmente diseñada. El edema de la mucosa en los senos frontal y maxilar se clasificó como: ninguna, leve ( $\mathrm{I}-2 \mathrm{~mm}$ ), más de $2 \mathrm{~mm}$ pero con aire central, u opacificación total del seno. El edema de la mucosa de los etmoides se clasificó como ninguna, leve, pronunciada 0 total. Luego clasificaron a los pacientes de acuerdo con el edema de la mucosa en las radiografías desde el grado $A$ al $D$. El grado A significaba que todos los senos paranasales eran normales, grado B significaba leve, grado C pronunciado y grado D opacificación total en al menos

un seno. Todas las radiografías fueron interpretadas por un solo radiólogo.

Con el fin de conocer el número mínimo de síntomas y hallazgos clínicos que en el primer examen del paciente podría dar información confiable sobre la aparición de edema de la mucosa visible radiológicamente, los resultados fueron estudiados con el análisis de factores discriminantes de acuerdo con Van der Geer ${ }^{(6)}$. El análisis incluyó los siguientes síntomas y hallazgos clínicos: secreción nasal purulenta (en nasofaringe, pus cubriendo el cornete inferior o el suelo de la nariz u obstrucción total de la nariz); dolor (dolor espontáneo o sensibilidad a la palpación de los senos); IRA durante las dos semanas previas a los síntomas reales; fiebre; neumonía o bronquitis; otitis media con efusión. Estos síntomas y hallazgos se comprobaron frente al edema de la mucosa evaluada radiológicamente.

Como resultados han obtenido una distribución de edad de los 175 pacientes incluidos en el estudio de niños de la siguiente manera: entre $0 \mathrm{y}$ 2 años (9 niños), entre 2 y 4 años (15 niños), entre 46 años (34 niños), entre 6 y 8 años (31 niños), entre 8 y 10 años (23 niños), entre 10 y 12 años (41 niños) y entre 12 y 15 años (22 niños). Con respecto a los hallazgos radiográficos de los pacientes los resultados fueron los siguientes: 48 niños (27\%) se clasificaron como Grado A, 30 niños (17 \%) como Grado B, 26 niños (15\%) como grado C y 71 niños (41\%) como Grado D.

Los síntomas y los signos clínicos confiables para la demostración radiológica del edema de la mucosa de

Revista Methodo: Investigación Aplicada a las Ciencias Biológicas. Facultad de Ciencias de la Salud.

Universidad Católica de Córdoba. Jacinto Ríos 571 Bo Gral. Paz. X5004FXS. Córdoba. Argentina. Tel.:

(54) 3514517299 / Correo: methodo@ucc.edu.ar / Web: methodo.ucc.edu.ar | DOI: 
Alegre G, Azar T, Marelli S, Pfleger M, Revol S, Cuestas E ¿El estudio radiográfico de senos paranasales es útil para confirmar el diagnóstico de sinusitis aguda?

los senos fueron: rinitis purulenta, IRA y dolor. En un análisis de los tres síntomas dominantes, denominados en lo sucesivo síntomas principales, cada paciente fue asignado a una de cuatro clases: clase 0 (sin síntoma principal), clase 1 (uno de los síntomas principales), clase 2 (dos de los síntomas principales) y la clase 3 (todos los síntomas principales presentes). Las correlaciones entre estas clases y los hallazgos radiológicos fueron: el $75 \%$ de los niños con al menos dos síntomas principales (clases 2 y 3 ) presentaron cambios radiológicos importantes (grado radiológico $\mathrm{C}$ o D), de los niños pertenecientes a las clases 0 y 1 , el $70 \%$ tenía los cambios radiológicos de grado A o $B$ y el $30 \%$ de los grados C y D. La mayoría de estos pacientes de grado $\mathrm{A}$ o B tenían condiciones alérgicas, como la bronquitis asmática y la rinitis alérgica.

Como conclusión definieron en el estudio analizado que el examen radiológico es innecesario si el paciente se presenta con la tríada clínica: IRA, secreción nasal purulenta y dolor, para el diagnóstico de sinusitis aguda.

En el escrito no consta si ha habido algún conflicto de interés con respecto a la realización de este estudio. Así como tampoco consta alguna fuente de financiación para llevar a cabo el estudio.

ANÁLISIS DEL ARTÍCULO
El diseño del trabajo no es específico para un estudio de diagnóstico, aunque con los datos que presenta pudo inferirse el valor diagnóstico de la clínica utilizando como patrón de referencia la radiografía de senos paranasales confirmada por la punción de los mismos.

El estudio incluye un número relativamente pequeño de niños que fueron seleccionados con todo el espectro de gravedad de la enfermedad (desde casos leves a casos severos).

Dado que todos los enfermos fueron sometidos a estudios radiográficos y bacteriológicos simultáneamente puede concluirse que no se ha incurrido en sesgos de verificación, aunque pueden existir sesgos de expectativa dado que no se menciona que los radiólogos y bacteriólogos hayan sido cegados a los datos clínicos. Tampoco se menciona la calificación y número de los radiólogos y bacterilógos, ni si se calculó la concordancia intra e interobservador, hecho este que potencialmente podría aumentar indebidamente la variabilidad de los resultados observados.

Para el análisis de la validez estadística del artículo debimos realizar un análisis secundario de los datos del mismo con el fin de definir los valores de la Sensibilidad (S), la Especificidad (E), el Valor Predictivo Positivo (VPP) y el Valor Predictivo Negativo (VPN), con sus respectivos IC95\%, a fin de

Revista Methodo: Investigación Aplicada a las Ciencias Biológicas. Facultad de Ciencias de la Salud.

Universidad Católica de Córdoba. Jacinto Ríos 571 Bo Gral. Paz. X5004FXS. Córdoba. Argentina. Tel.:

(54) 3514517299 / Correo: methodo@ucc.edu.ar / Web: methodo.ucc.edu.ar | DOI: 
Alegre G, Azar T, Marelli S, Pfleger M, Revol S, Cuestas E ¿El estudio radiográfico de senos paranasales es útil para confirmar el diagnóstico de sinusitis aguda?

aproximarnos a una conclusión más confiable. Para ello se definió como enfermos (sinusitis clínica) a los pacientes incluidos en la Clase 2 y 3 según los hallazgos clínicos y como sanos aquellos que se definieron como Clase 0 y 1 . Por otro lado se consideró a aquellos pacientes con radiografía patológica a los que presentaron un Grado C $\circ$ D de la misma y normal a aquellos con Grado A o B.

Teniendo en cuenta que el $75 \%$ de los niños se encontraban clasificados en la Clase 2 y 2 según la clínica y que el $70 \%$ de los niños clasificados como Clase 0 - 1 presentaban radiografías de Grado A o B y el $30 \%$ restante Grado C o D, para realizar el análisis de los datos se desarrolló la tabla 1.

Tabla 1. Población incluida en el trabajo analizado

\begin{tabular}{|c|c|c|c|}
\hline & $\begin{array}{c}\text { Clase } \\
\text { II o III } \\
(+)\end{array}$ & $\begin{array}{c}\text { Clase } \\
0 \text { o l }(- \\
\text { ) }\end{array}$ & Total \\
\hline $\begin{array}{l}\text { Rx } \\
\text { Grado } \\
\text { C o D } \\
(+)\end{array}$ & 73 & 24 & 97 \\
\hline $\begin{array}{l}\text { Rx } \\
\text { Grado } \\
\text { A o B (- } \\
\text { tiaídos }\end{array}$ & 23 & 55 & $\begin{array}{r}78 \\
\text { iores }\end{array}$ \\
\hline $\begin{array}{l}\text { Total } \\
\text { ccedio }\end{array}$ & $\begin{array}{l}96 \\
905\end{array}$ & $\begin{array}{l}79 \\
\text { cátculos }\end{array}$ & $\begin{array}{l}175 \\
\text { de }\end{array}$ \\
\hline
\end{tabular}

sensibilidad, siendo esta de un $76,04 \%$ con un intervalo de confianza del 95\% (IC $95 \%$ ) de 66,61 -83,47\%; de especificidad, la cual fue de $69,62 \%$ (IC 95\% de 58,77-78,66\%); del VPP, siendo de $75,26 \%$ con un IC $95 \%$ de 65,82 -
82,77\%; y del VPN, el cual dio un valor de $70,51 \%$ (IC 95\% 59,62-79,16).

El cociente de probabilidad de una prueba positiva fue de $2,5(2,28-2,73)$ y para una prueba negativa de $0,34(0,31$ $0,38)$; con una prevalencia de sinusitis aguda de 55,43 (47,78-63,08).

\section{COMENTARIOS}

En la actualidad es muy frecuente observar, sobre todo en las guardias pediátricas, la solicitud de radiografías de senos paranasales con el fin de diagnosticar sinusitis aguda, y así justificar el uso de antibióticos. Por lo cual nos pareció pertinente la necesidad de realizar la búsqueda de evidencias publicadas, la cual justifique o no esta práctica, dada la irradiación a que son sometidos los pacientes, la pérdida de tiempo y la utilización inadecuada de los recursos.

Como se ha comentado previamente solo se ha encontrado un estudio que analiza este tema en la población pediátrica, con algunas limitaciones ya que solo presentaba resultados meramente descriptivos de los datos recolectados. Tras lo cual debimos realizar un análisis estadístico con dichos datos para obtener los valores de S, E, VPP y VPN, y así aproximarnos a una conclusión más firme.

Luego de este análisis pudimos concluir que hasta el momento no existe evidencia que avale dicha práctica, ya que de los pacientes enfermos sólo 7 de

Revista Methodo: Investigación Aplicada a las Ciencias Biológicas. Facultad de Ciencias de la Salud.

Universidad Católica de Córdoba. Jacinto Ríos 571 Bo Gral. Paz. X5004FXS. Córdoba. Argentina. Tel.:

(54) 3514517299 / Correo: methodo@ucc.edu.ar / Web: methodo.ucc.edu.ar | DOI: 
Alegre G, Azar T, Marelli S, Pfleger M, Revol S, Cuestas E ¿El estudio radiográfico de senos paranasales es útil para confirmar el diagnóstico de sinusitis aguda?

cada 10 presentan una radiografía positiva, implicando que 3 no serán tratados, aumentando la probabilidad de complicaciones (especialmente celulitis preseptal) y que de los pacientes sanos sólo 7 de cada 10 presentan una radiografía normal, implicando que 3 serán tratados innecesariamente.

\section{RECOMENDACIÓN FINAL}

Por el momento, con la evidencia disponible no se recomienda el estudio radiográfico con placas de los senos paranasales en niño para el diagnóstico de sinusitis aguda. Con los datos extraídos puede afirmarse que las radiografías simples de senos paranasales no solo no son de utilidad sino que muchas veces confunden más de lo que ayudan. Teniendo en cuanta los datos clínicos de infección respiratoria alta de más de dos semanas de evolución, rinitis purulenta, y dolor, se puede definir razonablemente el diagnóstico y prescribir el tratamiento indicado. Cuando existan dudas diagnósticas o fallo del tratamiento deberá indicarse Tomografía Computada.

\section{INFORMACIÓN ADICIONAL (1)}

Las radiografías simples de los senos se utilizaron profusamente en el pasado, pero la comprobación de su escasa sensibilidad y especificidad en la detección de la sinusitis ha motivado que su práctica cada vez sea menos recomendada. Entre un 30 y un $50 \%$ de las radiografías simples de los senos realizadas en niños asintomáticos son anormales. Además, alrededor del $45 \%$ de los pacientes con sinusitis demostrada por TC presentan radiografías simples dentro de la normalidad. El estudio de imagen preferido en la valoración de los senos paranasales es la TC, preferentemente en plano coronal y sólo cuando se pretenda descartar una complicación extrasinusal. Es la única prueba capaz de valorar la anatomía de todos los senos, incluyendo el complejo osteomeatal, y es muy superior a la radiografía simple en el diagnóstico de la sinusitis. Entre sus inconvenientes se incluye la duración de la exploración, que se va reduciendo con los avances tecnológicos, la dosis de radiación y el coste. Su elevada sensibilidad, derivada de su capacidad para detectar mínimos cambios en la mucosa, se acompaña de una baja especificidad que genera problemas de sobrediagnóstico, al detectar alteraciones sin significación clínica. De hecho, un $40 \%$ de los niños que han padecido en las últimas 6 semanas un proceso viral de la vía respiratoria superior presentan TC anormales, así como un $15 \%$ de la población infantil en general. La especificidad es más baja cuanto menor es la edad del paciente, debido al menor desarrollo de los senos. Por lo cual su uso se recomienda en la valoración 
Alegre G, Azar T, Marelli S, Pfleger M, Revol S, Cuestas E ¿El estudio radiográfico de senos paranasales es útil para confirmar el diagnóstico de sinusitis aguda?

preoperatoria de la sinusitis crónica y

para descartar complicaciones en la

sinusitis aguda en aquellos casos de

\section{BIBLIOGRAFÍA}

1. Garcia Ferandez A. Sinusitis infantiles. An Pediatr, Monogr. 2003;1(1):35-9.

2. Wald ER., Applegate KE, Bordley C, Drrow DH. Clinical Practice Guideline for the diagnosis and Management of Acute Bacterial Sinusitis in Children Aged 1 to 18 Years. Pediatrics. 2013;132:e262e280.

3. Engels EA, Terrin N, Barza M, Lau J. Meta-analysis of diagnostic tests for acute sinusitis. J Clin Epidemiol. 2000;53(8):852-862. fracaso terapéutico o empeoramiento de los síntomas. ${ }^{(1)}$
4. Jannert M, Andréasson L, Helin I, Pettersson $H$. Acute sinusitis in children - symptoms, clinical findings and bacteriology related to initial radiologic appearance. International Journal of Pediatric Otorhinolaryngology. 1982; 4:139148.

5. Alexsson A, Jensen C. The reontgenologic demostration of sinusitis. AJR. 1974; 122:621-627.

6. Van der Geer JP. Introduction to Multivariante Analsis for the Social Science, Witt-Freeman, San Francisco, CA, 1971, pp. 184-191. 